\title{
Influence of the microstructure on the strain of micro components
}

Received: 28 June 2005 / Accepted: 7 November 2005 / Published online: 7 February 2006

(C) Springer-Verlag 2006

\begin{abstract}
In order to ensure the reliable dimensioning of micro components the influence of the material anisotropy of grains upon the local stress under load is investigated. Based on a point process a program for the generation of micro components with their threedimensional microstructure for the finite element analysis has been developed, in order to determine the strains in micro components which have only few grains in critical areas. The limitation of the finite element analysis with isotropic material models and the necessary consideration of the microstructure have been investigated in detail. Now it is possible to provide designers with additional knowledge to design efficient and reliable micro components.
\end{abstract}

\section{Introduction}

Modern product development processes are more and more dependent on time, cost and quality restrictions. Therefore the use of numerical methods for the simulation of real component behaviour becomes increasingly important. Especially the finite element method (FEM) is used to substitute or at least reduce the number of cost intensive physical tests. FEM serves to create components virtually, calculate strains, realize dimensioning, and compare alternatives before actually generating a first preliminary physical prototype.

Micro technology also employs numeric methods like FEM in order to save time and money. However, micro technology deals with dimensions that are sensitive to certain effects which can be neglected in other fields.

A. Albers $(\bowtie) \cdot$ D. Metz

IPEK-Institute of Product Development Karlsruhe,

University of Karlsruhe (TH), Kaiserstr. 10,

76131 Karlsruhe, Germany

E-mail: cae@ipek.uni-karlsruhe.de

Tel.: + 49-721-6082371

Fax: + 49-721-6086051
This fact raises the question whether the FEM can be employed in this field unconditionally. Hence, this paper deals with the problem of a reliable simulation of micro components by using the FEM and the transferring macroscopic modeling to micro technology.

\section{Modeling of the microstructure}

The collaborative research center (SFB) 499 "Development, Production and Quality Assurance of Primary Shaped Micro Components from Metallic and Ceramic Materials" aims at applying production processes of primary shaping, i.e. microPIM and micro casting, to the medium and large-scale production of three-dimensional miniature components out of metal and ceramics (Löhe et al. 2003). Not only the production process has to be investigated, but also the layout and design must be analyzed. The development methodology is being investigated (Albers et al. 2003) and experiments regarding the characterization of material are being realized (Beck et al. 2003); simultaneously numeric analysis are taking place that specifically investigate how the strain in polycrystalline micro components is modified. The configuration of the microstructure has to be modeled for this experiment.

\section{Modeling of the grains}

In order to model the microstructure, a linear-elastic material is used to describe the material behavior of the grains. Furthermore, the grains are assumed to be free of defects so that the characteristics of the material can be described with mono-crystal parameters. The collaborative research center 499 uses the so-called Stabilor G. Since no mono-crystal data is available for this material, the mono-crystal data of gold were applied to it. Other materials being utilized are 17-4 PH as sinter steel and zirconia as sinter ceramic. Irregular poly-crystals are most commonly modeled with a Voronoi tessellation 
(Stoyan et al 1995). As a starting point a desired number of randomly generated points is taken. This point generating process is chosen in a way that enables the Voronoi tessellation to describe the microstructure of the polycrystals as realistically as possible, possessing also simple statistic properties. A Voronoi tessellation is generally understood as a tessellation of a region into convex polyhedrons, which do not overlap and consume the whole area (Fig. 1). Owing to the properties of a polycrystal, it is reasonable to use only a homogeneous and isotropic point process, such as the Poisson process (Stoyan and Stoyan 1992; Okabe et al. (1992).

To model the microstructure of the materials which are used by the collaborative research center, a hardcore point field is used in order to control the minimum distance between neighboring points. This way, the grains have a globular shape. By changing the number of points, the grain size is varied proportionally to characteristic component dimensions. These numerically generated models are validated with micrographs.

\section{Modeling of the material}

Two assumptions are made when modeling the material behavior of a polycrystal: At first, the polycrystal is macroscopically isotropic, i.e. a preferred direction (texture) of the crystallographic axes of the single grains does not exist; secondly, the orientation of adjacent grains is independent. To describe the orientation of the crystals, the three Eulerian angles are used (Bunge 1982). For the FEM calculation, each grain is given a random orientation from the anisotropic single crystal values. The Eulerian angles are used as random variables here.

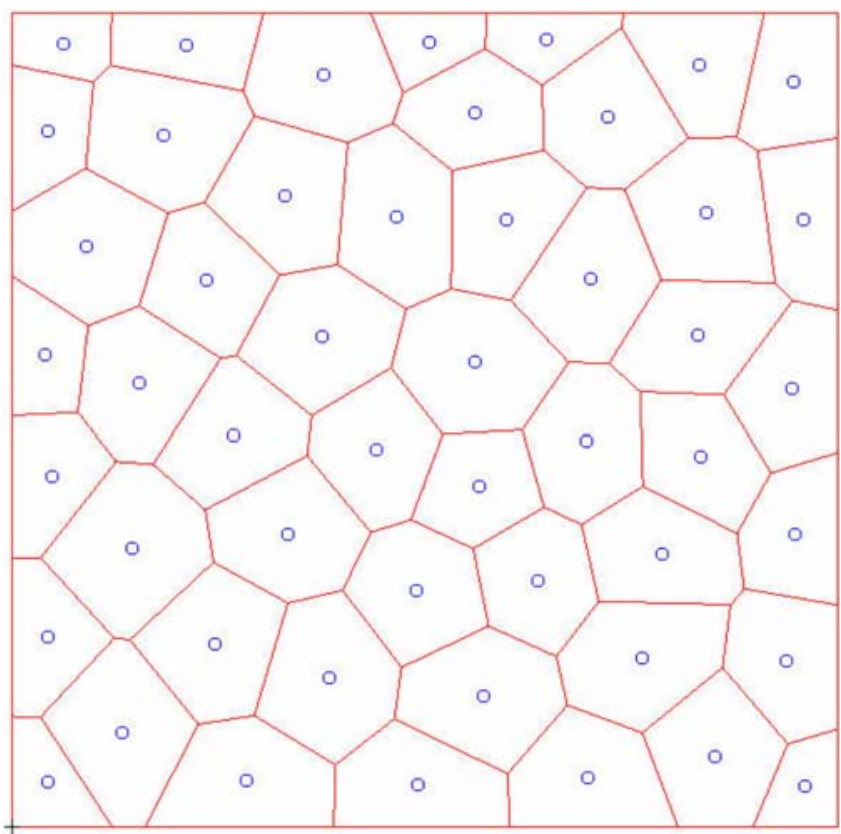

Fig. 1 Voronoi tessellation of a two-dimensional set of points
Material input files with isotropic results and anisotropic material input files in which the material is oriented randomly are allocated in the FEM analysis for an arbitrary number of grains.

\section{Finite element mesh}

The geometry of the grains is meshed with the pre/post processor MSC.Patran. For plane Voronoi tessellations, an automatic meshing by means of the Patran command language (PCL) can be realized by generating a surface which is based on the vertices of the Voronoi tessellation (Weyer et al 2002). Afterwards the finite elements describing a grain are summarized as a group, and a random material orientation will be allocated to this group for the calculation with the finite element code Abaqus. The two-dimensional approach is sufficient for simple micro components or specimens like tensile specimens. However, as will be shown below, the two-dimensional approach is not sufficient for micro components that are more complex; in this case a three-dimensional model is required. The meshing as described is not suitable in the three-dimensional case because of the complex grain structure and the generation of the mesh. Another procedure is more practical in this case: A software tool which has been developed at IPEK generates the micro component and the interior is provided with a three-dimensional random set of points. Shape and the number of the grains can be varied by changing the distance of adjacent points. Afterwards the micro component is generated with the Patran command language in MSC.Patran and is meshed without taking into account any grain geometry, as is common in the macroscopic domain.

Now the micro component has been meshed and can be calculated isotropically. Thereafter, MSC.Patran is used to determine the center of gravity of the finite elements. These centers of gravity are imported into the IPEK program again and will now allocate the finite elements to the random set of points. The finite elements describing a grain will then be summarized as a group. As described above a random material orientation is allocated to this group for the analysis with Abaqus.

As can be seen in Fig. 2, the grains are not described exactly in this case. However, different meshed sample geometries have shown that the results depend on the mesh to a minor degree only (see Fig. 3). Model 1 has a link with an exact description of the grain geometry (compare Fig. 2, left). Model 2 contains approximately the same number of finite elements, but with an isomesh (compare Fig. 2, right). An isomesh was also used in model 3 and 4; however contain four respectively sixteen times more finite elements than model 2.

Figure 3 compares the strain energy affecting all models. Because of its distinctive anisotropic behaviour zirconia was used as material. Each model has been analysed isotropically once and then 50 times with 
Fig. 2 A two-dimensional Voronoi tessellation as sample geometry with automatic meshing (left) and simple meshing (Isomesh) for the three-dimensional use (right)
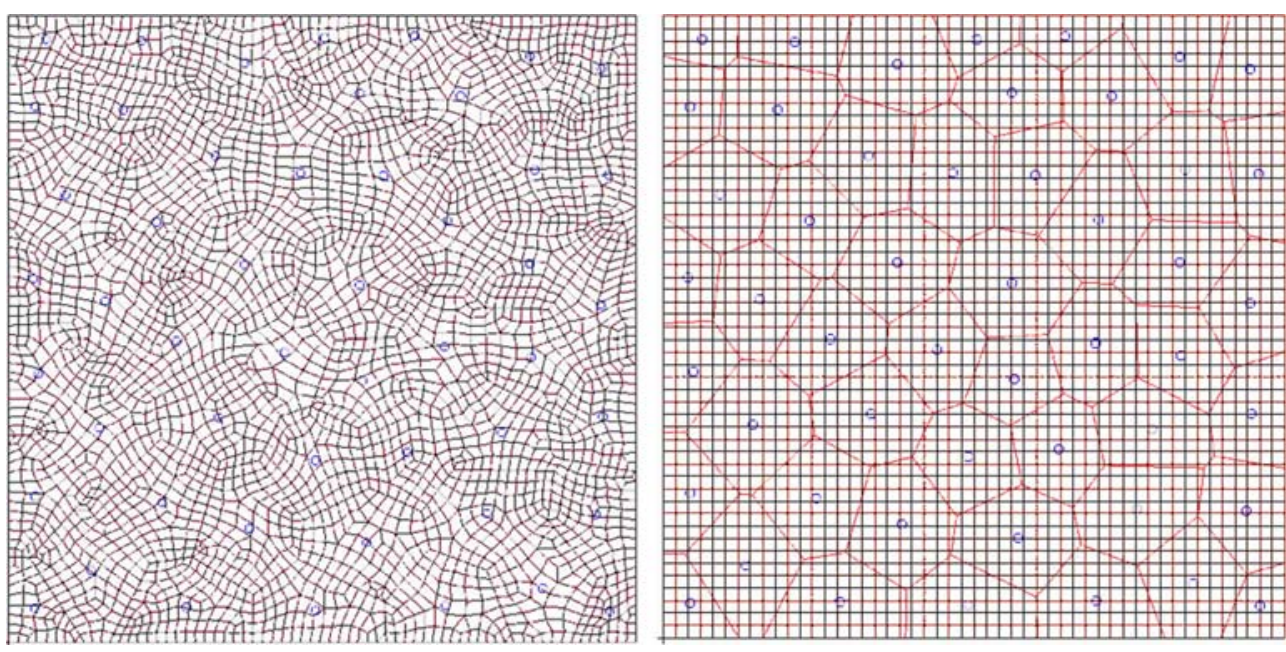

Fig. 3 Comparison of different meshes

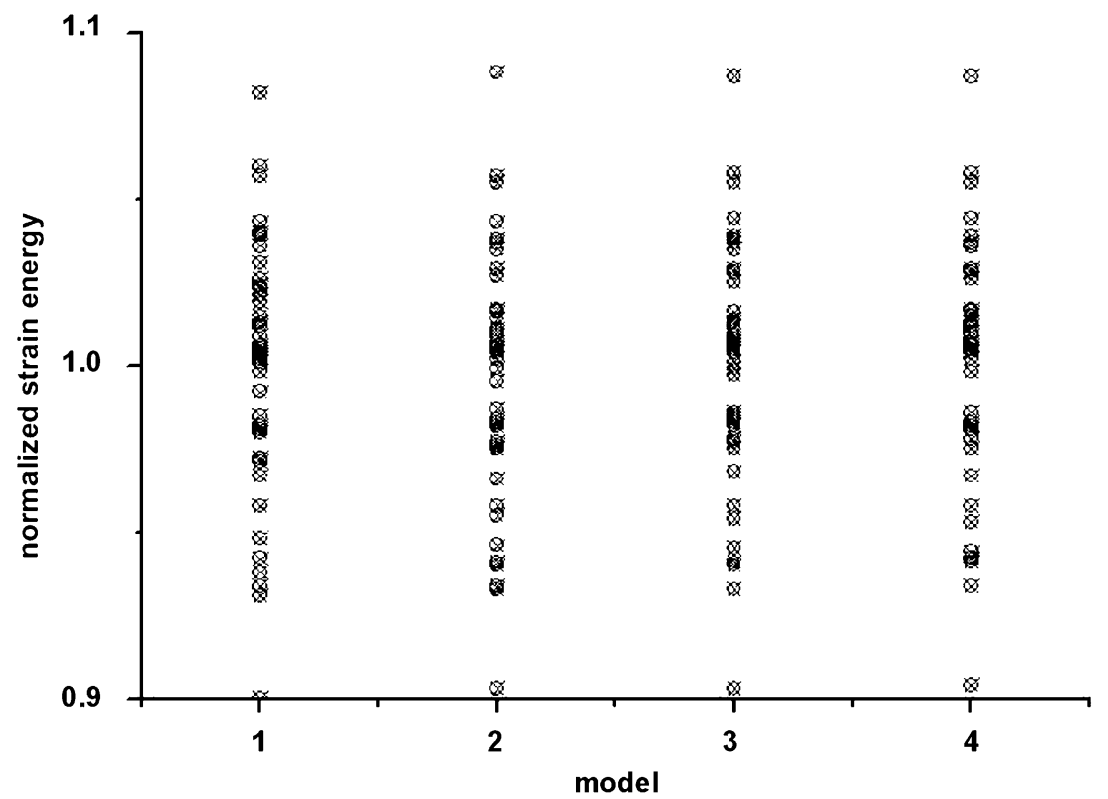

different anisotropic material distribution. The anisotropic results have been normalized by means of their average value.

In the isotropic case, the results of all models coincide. The average values of the anisotropic calculations increase by $13 \%$ in comparison to the isotropic results. Furthermore, the average values of models $2-4$ show a maximum fluctuation of $0.3 \%$ in comparison to the average value in model 1 . The standard deviation of anisotropic calculations adds up to $4 \%$ in all models. Studying single anisotropic material distributions, the strain energy deviated at most $1.3 \%$ in the models $2-4$ in comparison to model 1. The analysis of the stresses shows similar results (Fig. 4).

As a result it can be concluded that single calculations with the same anisotropic material files fluctuate slightly, depending on the two meshing methods introduced above, which can be neglected. This is especially true due to the fact that the fluctuations vanish within the context of the required stochastic analysis. The procedure of meshing with an isomesh introduced above is an dimensional microstructures.

\section{Results}

Due to the dimensions of the micro components and specimens with critical cross sections of 100-200 $\mu \mathrm{m}$ and the grain size of zirconia with $0.3-1 \mu \mathrm{m}$, conventional methods of analysis and an isotropic modeling of the ceramic material can certainly be applied.

However, the influence of the anisotropy of the grains in metallic materials with regard to the current dimensions and in ceramics if the micro components are miniaturized even more has not been determined yet.

In the following the possibility of an application of the FEM on micro dimensions will be described. appropriate alternative and allows the study of three- 
Fig. 4 Von Mises stresses in different meshes (left model 1, right model 2) but with the same anisotropic material distribution
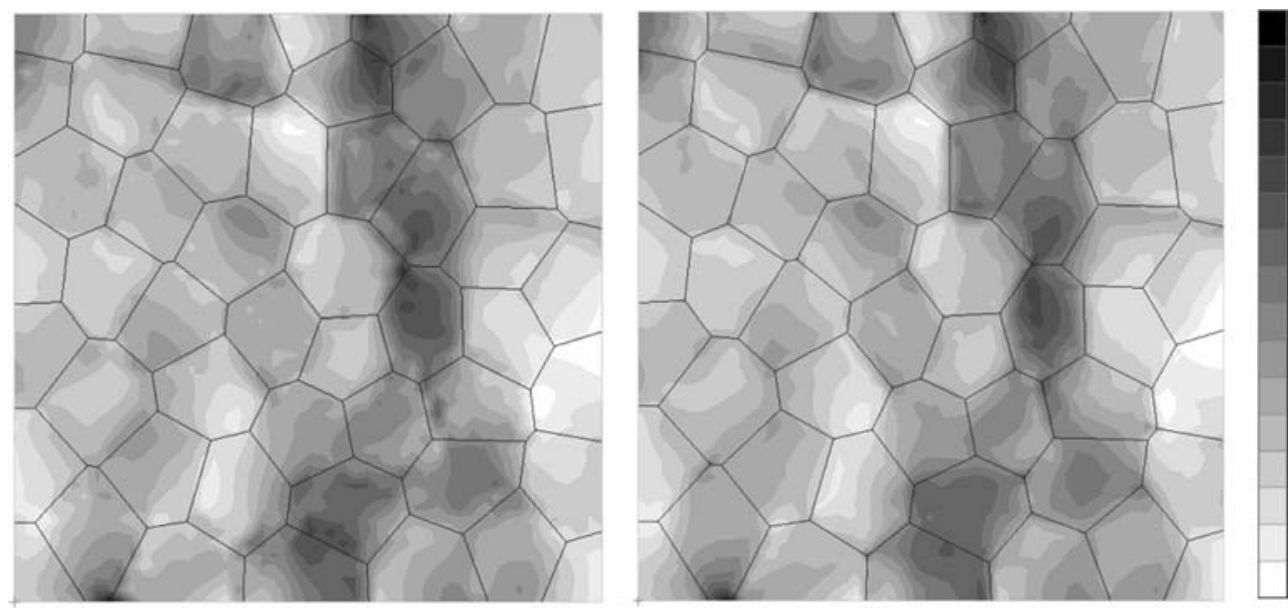

Afterwards complex micro components containing only few grains in critical parts of the components will be examined.

\subsection{Tensile specimen}

The following results show the limits of isotropic modeling. In analogy to the micro tensile tests realized by the collaborative research center, ten two-dimensional tensile specimen were modeled, each $1,000 \mu \mathrm{m}$ long and $200 \mu \mathrm{m}$ wide, with varying numbers of grains. The number of grains of the tensile specimen varies from 10 to 250 , the number of grains in the critical cross-section are in a range from 1 to 8 . The tensile test was modeled linear-elastic with zirconia, 17-4 PH and gold. Figure 5 demonstrates the strain energy of 100 anisotropic calculations per model for zirconia, normalized by their average value.
As can be observed, about the average value the scatter reduces with increasing numbers of grains. The standard deviation decreases continuously from 8.8 to $2.1 \%$. The same qualitative behaviour can be observed with 17-4 PH and gold; the scatter, however, is less for these materials. By means of this investigation we can estimate that quasi-isotropic conditions can be assumed if the number of grains in the cross-section exceeds a value of eight.

\subsection{Micro components}

Results of specimen with simple geometries and load cases cannot be transferred without further examinations to real components. Therefore, the influence of the microstructure has also been tested on concrete micro components, in this case the tooth of a micro gear. In analogy with the analysis of the tensile specimen, micro
Fig. 5 Scatter of strain energy versus the number of grains

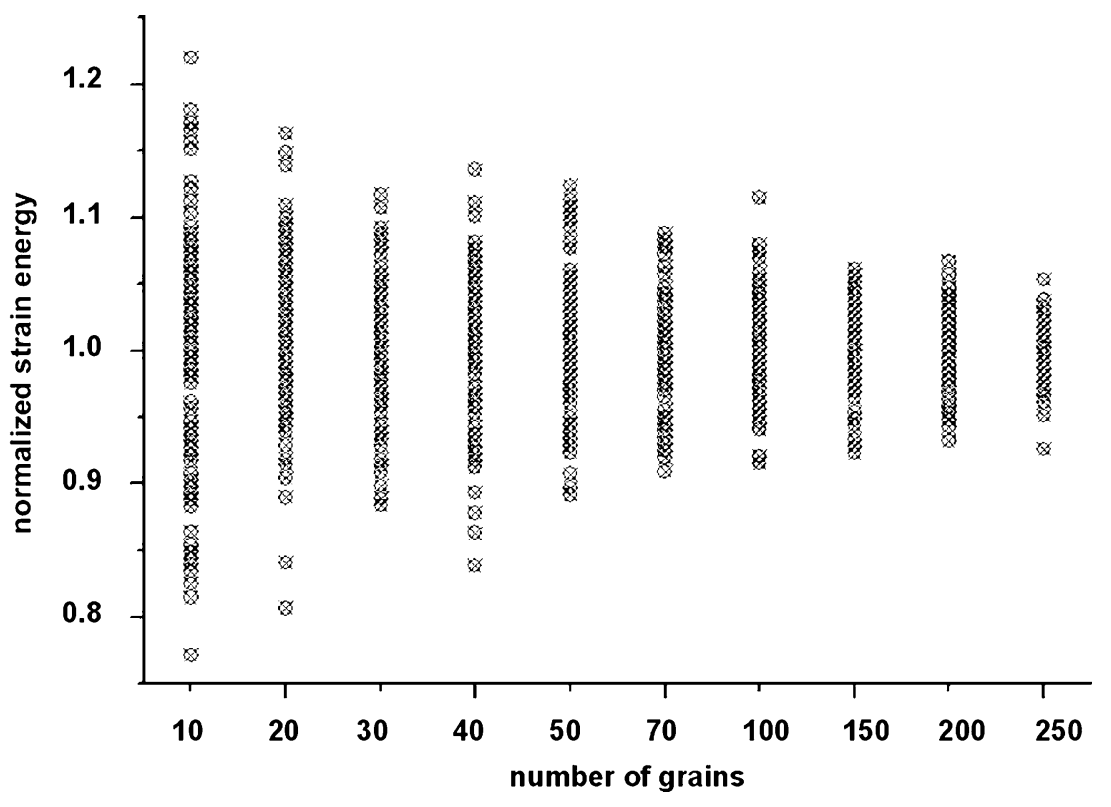



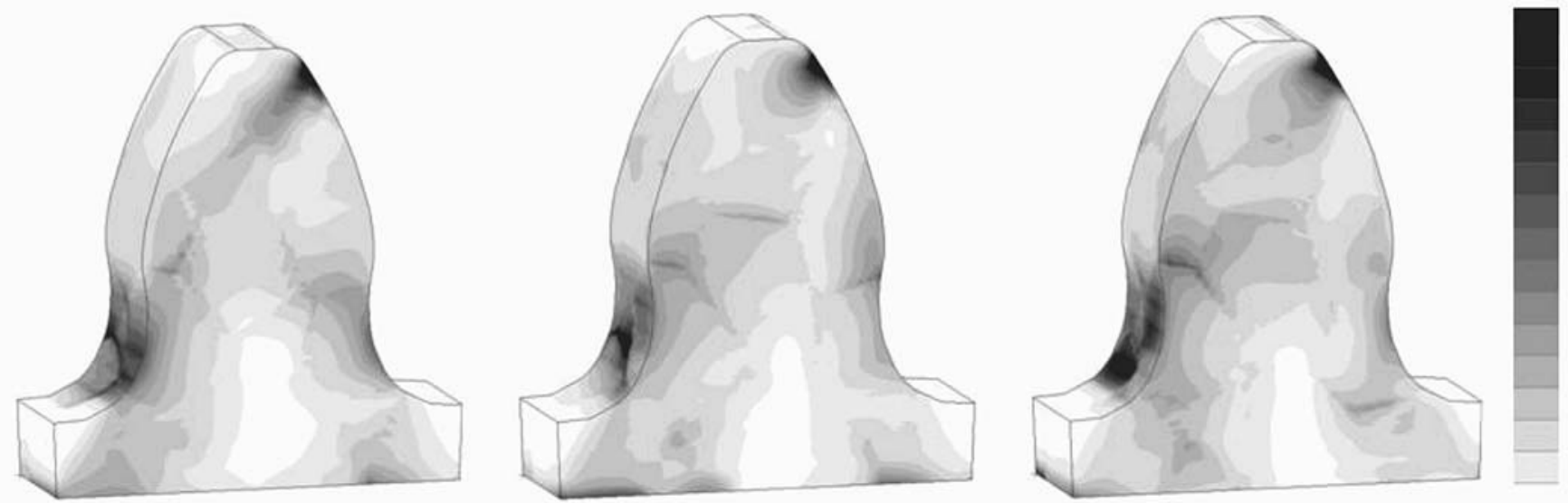

Fig. 6 Von Mises stresses in a loaded micro tooth as a result of different anisotropic material distributions

components were also modeled two-dimensional. The analysis made clear that in critical areas it is necessary to take into account the microstructures when dimensioning micro components with few grains (Albers and Metz 2003). Due to the two-dimensional structure, however, the limits of the isotropic calculation and the stress in the tooth root caused by the anisotropy of the grains could only be determined by consulting the results of the anisotropically simulated tensile specimen.

For this reason, a special software application has been created. It generates three-dimensional micro components with their microstructure which can then be calculated by means of the FEM. For the analysis, a tooth of the micro planetary gear train that belongs to demonstrator system of the collaborative research center was modeled with a varying number of grains. The models were then loaded with a force on the flank (Fig. 6).
Four models with $30,70,150$ and 450 grains respectively were generated. This is approximately equivalent to a number of 4 to 16 grains at the tooth root. Figure 7 shows how in zirconia the maximum von Mises stresses scatter at the tooth root with regard to the average value of the anisotropic calculations.

With an increasing number of grains at the tooth root, the scatter decreases around the average value. The standard deviation lies between 14.6 and $10.7 \%$. In the worst case scenario, the stress increased by $34.5 \%$ with respect to the average value. The increase is reduced to a maximum of $27 \%$ due to a higher number of grains at the tooth root. Similar results can be shown for 17-4 PH and gold.

The models described have not fully achieved a quasiisotropic state yet; therefore experiments with larger grain numbers in the tooth base are being investigated in future. However, results for the dimensioning of
Fig. 7 Scatter of the maximum von Mises stresses in the tooth root depending on the number of grains

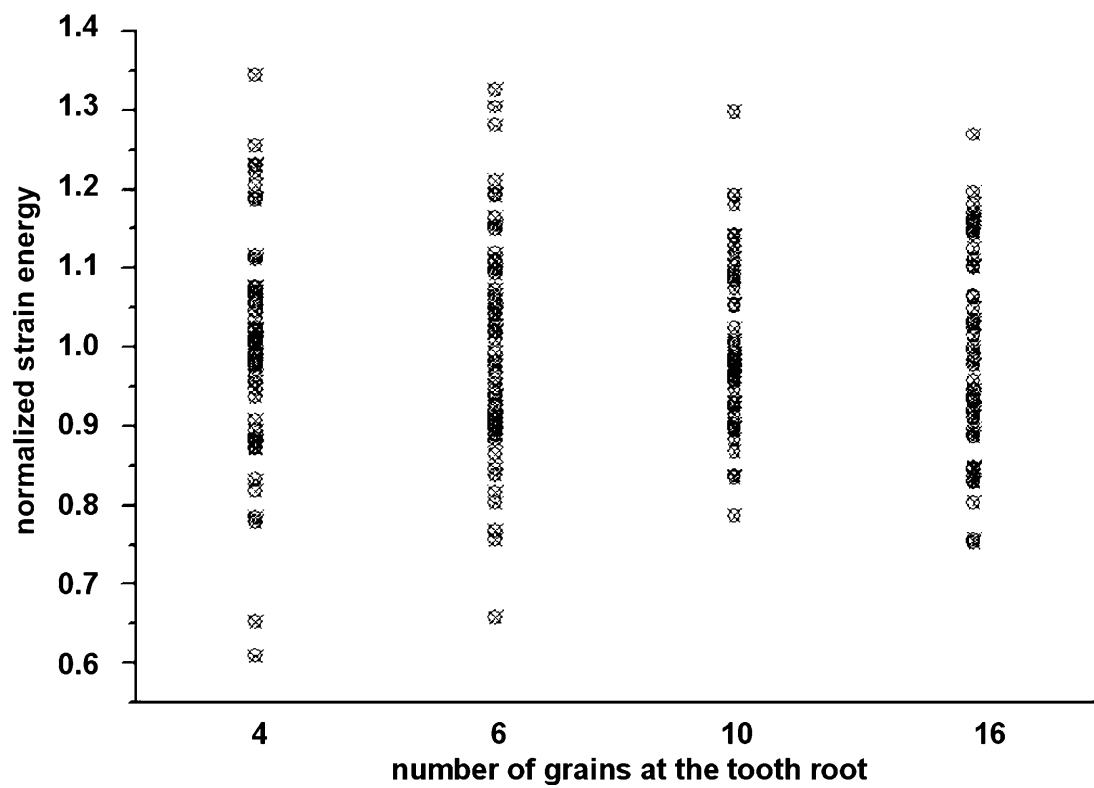


complex micro components with small numbers of grains in critical areas can already be determined.

\section{Conclusion}

The investigations presented in this paper have demonstrated the influence of the microstructure on the stresses of micro components and the limits of the FEM with regard to isotropic calculation in the micro regime. By means of the developed program for the generation of three-dimensional microstructures for the finite element analysis, it is now possible to determine strains on micro components which have only few grains in critical areas. This enables a reliable dimensioning of those micro components due to the consideration of the relevant anisotropic material behaviour of the grains.

Acknowledgements This work is supported by the German Research Foundation (DFG) within the collaborative research center (SFB) 499 "Development, Production and Quality Assurance of Primary Shaped Micro Components from Metallic and Ceramic Materials"

\section{References}

Albers A, Metz D (2003) Investigations on the limitations of the feanalysis of micro-devices. In: Proceedings of the 4th international conference, European society for precision engineering and nanotechnology, pp 121-124, 19.05-20.05, Aachen

Albers A, Burkardt N, Marz J (2003) Restrictions in the design of gear wheel components and drives for micro technology. Microsyst Technol 9(3):192-196

Beck T, Schneider J, Schulze V (2003) Charakterisierung und Prüfung von Mikrobauteilen, Kolloquium Mikroproduktion, ISBN 3-8027-8670-X, S 171-182

Bunge HJ (1982) Texture analysis in materials science. Butterworth, London

Löhe D, Spath D (2003) Mikrourformen-Der SFB 499; Kolloquium Mikroproduktion, Februar ISBN 3-8027-8670-X, S 3343

Okabe A, Boots B, Sugihara K (1992) Spatial tesselations - concepts and applications of Voronoi diagrams. Wiley, New York

Stoyan D, Stoyan H (1992) Fraktale Formen Punktfelder. Akademie-Verlag

Stoyan D Kendall WS, Mecke J (1995) Stochastic geometry and its applications, 2nd edn. Wiley, Chichester

Weyer S, Frölich A, Riesch-Oppermann H, Cizelj L, Kovač M (2002) Automatic finite element meshing of planar Voronoi tsselations. Eng Fract Mech 69:945-958 